\title{
Neue Arzneimittel, Spezialitäten und
} Geheimmittel.')

Von Dr. Franz Zernik in Steglitz.

XII.

Ein echtes Kurpfuschermittel, das der nördlichen Rheinprovinz entstammt, ist Anton Ambraus Wassersuchtsmittel. Die nach dem Umschütteln grau-gelblich-trübe Flüssigkeit besitzt den charakteristischen Geruch sauer gewordenen Kleisters und widerlichen Geschmack. Die chemische Untersuchung bestätigte, daB hier das Produkt einer sauren Gärung kleberhaltiger Stoffe vorlag - vermutlich ein verdorbener Weizenmehlkleister.

Borau-Sommersprossencream enthält nicht, wie der Name anzudeuten scheint, harmlosen Borax, bzw. Borsäure, vielmehr je $5 \%$ Hydrargyrum praecipitatum album und Bismutum subnitricum, verrieben mit einer parfümierten, etwas Wachs und Wasser enthaltenden Paraffinsalbe. Die Tube zu $20 \mathrm{~g}$ Inhalt kostet 1 Mark.

Professor Max Danas Mittel gegen Asthma, eines der vielen Präparate, mit denen England den von derartigen Mitteln doch wahrlich schon genug überschwernmten deutschen Markt beglückt, ist ein Räucherpulver, das sich von den sonst gebräuchlichen nur insofern unterscheidet, als es neben Salpeter (50\%), Stramonium und Lobelia auch noch Thea nigra enthält.

Unter dem Namen Epileptol (früher Antiepilepticum Dr. Rosenberg) bringt die Admiral-Apotheke in Berlin ein Epilepsiemittel in den Handel. das der Erfinder und Darsteller, Dr. med. J. Rosenberg in Berlin, als "Acidum amidoformicicum condensatum " bezeichnet. Dieser Name ist irreführend und nichtssagend, denn eine ihm entsprechende verbindung kennt die Chemie überhaupt nicht. Die chemische Untersuchung des Mittels ergab vielmehr. daß es besteht in einer etwa 30\%igen wäßrigen Lösung enes geringe Mengen Hexamethylentetramin entbaltenden Gemisches aus Formamid mit einer Verbindung von Formaldehyd mit Formamid. Die Gesamtmenge des - sehr leicht abspaltbaren Formaldehyds im Epileptol beträgt rund $4 \%$. Ueber praktische $\mathbf{E r}$ fahrungen mit dem Mittel ist außer zwei Arbeiten aus der eigenen Feder des Erfinders und Darstellers in der medizinischen Presse bisher nichts veroffentlicht worden. Preis: $100 \mathrm{~g} 7 \mathrm{Mark}$. bzw. $200 \mathrm{~g} 14$ Mark.

1) VgI diese Wochenschnift 1946, No. 36, 43, 49, 52, 1907, No. 4, 12, 18, 23. $27,42$. 
Im Jahre 1905 wurde unter dem Namen Lenicet ein schwerlösliches, baqisches Aluminiumacetat für therapeutische Zwecke empfohlen ale Ersatz des gebrduchlichen Liyuor Aluminii acetici. vor dem es dop Vorzug des festen Aggregatzustandes hat. Zu Beginn des laufenden Jahres brachten die Chemischen Werke Fritz Friedlunder G. m. b. H. in Berlin abnliche Praparate in den Handel nämlich Fston, nach Angabe der Darsteller ein schwerlösliches Aluminium $1 / 3$-Acet at, Subeston, Aluminium $1 / 2$-Acetat, und Formeston, schwerlösliches, basisches Aluminiumformiat-Acetat -. alles angeblich Pruparate von genall präzisierter und formulierter chemischer Zusammensetzung. Während Lenicet ein üußerst staubfeines Pulver darstellt, dessen Zusammensetzung den Angaben des Darstellers im wesentlichen entspricht. besitzen die drei anderen Mittel einen weit geringeren Feinheitsgrad - die betreffende Patentanmeldung betrachtet diese geringere Feinheit sonderbarerweise als Vorzug auBerdem alle einen Gehalt von rund 11\% Aluminiumsulfat, zweifellos infolge Verwendung eines stark basischen Aluminiumsulfats als Ausgangsmaterial - entgegen den Angaben der Patentanmeldung. $\mathrm{DaB}$ diese Beimengung im vorliegenden F'alle unzulässig ist, liegt auf der Hand, denn der Vorzug jener Präparate soll gerade darin besteben, daA sie infolge ihrer schweren Löslichkeit nur allmălich adstringierend wirken; Aluminiumsulfat ist aber, wie Alaun, leicht losslich und wirkt dementsprechend energischer. Die Darsteller behaupten zwar gegenüber den obigen Feststellungen, daß unglticklicherweise gerade Proben untersucht worden seien, die ubsichtlich einen Zusatz von Aluminiumsulfat erhalten hätten; ganz abgesehen aber davon, daß ein solcher Zusatz kaum verständlich wäre, muß dem entgegengehalten werden, $\mathrm{da} B$ auch in von anderer Seite untersuchten Proben stets Aluminiumsulfat gefunden wurde.

Aus Italien kommt Filodentol (Carlo Erba in Mailand), ein Zahnschmerzmittel das nicht, wie gewöhnlich, mit dem erkrankten Zahn oder dessen Umgebung in Berührung zu bringen ist, vielmehr soll das Mittel aus der gehöhlten Hand in das dem schmerzenden Zahn nicht benachbart gelegene Nasenloch aufgezogen werden, worauf dann jeder Schmerz sofort verschwindet. Mit Rücksicht auf diese prompte Wirkung wahrscheinlich wird für ein kleines, $5 \mathrm{~g}$ enthal. tendes Fläschchen mit einer rotgefärbten. schwachen Lösung von atherischen Oelen (Pfefferminz und Zimt) in verdünntem Weingeist, der Preis von 1 Mark verlangt!

Dr. Kellers nerven- Ind blutstärkender Kräntertee (Apotheker P. Baessgen in Dortmund) kommt in den Handel in Kaitons, enthaltend sieben abgeteilte Portionen zu rund je $20 \mathrm{~g}$. Preis 2,60 Mark Eine Portion mit drei Tassen kochenden Wassers aufgebrüht, gilt als Tagesdosis. „Bei hartnäckigen Leiden muB der Tee beharrlich weiter getrunken werden, bis vollständige Heilung eingetreten ist“ etc. Die Zusammensetzung des Tees an sich ist nicht ungeschickt; er besteht aus 10 Teilen Rad. Valerian., 8 Teilen Kal. bromat. cryst., 5 Teilen Cort. Chinae, 4 T'eilen Fruct. Papav. matur. und je 1 Teil Rad. Liquirit., Fol. Sennae und Fol. Menthae piperit.

Ganz hervorragende Wirkungen schreibt die chemische Fabrik "Der Linden" in Kirchberg-Wolfersgrün (Sachsen) ihrer Hautpasta Pheun zu. Diese entfernt nämlich bei einfachem, leichtem Auftragen auf die Haut nicht nur den Schmutz. sondern sie tötet auch die in den untersten Höhlungen der Haut ver"borgenen Krankheitskeime vollkommen und sicher ab. „Lästige Hautflecke, Röte, Hautausschlige, aufgesprungene Hände, leichte Hautentzündungen verschwinden nach ganz kurzem Gebrauch." Trifft das alles zu, so mäBte der $\mathrm{P}_{1}$ eis von $1 \mathrm{Mark}$ für die $25 \mathrm{~g}$ enthaltende Tube als mäbig bezeichnet werden. Die chemische Untersuchung des Mittels eröffnet freilich ein weniger gthnstiges Prognostikon; nach ihr enthielt die Paste, eine weiße, purfümierte, salbenartige Masse, neben rund $31 \%$ Weichparaffin und $5 \%$ Wasser nur $10 \%$ eines schleimgebenden Trockenstoffes und $2 \%$ Seife. 